\title{
Empoderamiento del estudiante frente a su aprendizaje a partir de secuencias didácticas
}

\section{Student empowerment in his learning from didactical sequences}

\section{Befähigung des schülers für seine ausbildung durch didaktische sequenzierung}

\author{
Myriam Ruth Posada de Ceballos ${ }^{1}$, Isabel Cristina Gutiérrez de Dussán ${ }^{2}$, María de los Ángeles Rivera Ortiz ${ }^{3}$
}

\section{Resumen}

En el presente documento se pretende demostrar la manera como una Secuencia Didáctica (SD), utilizada como categoría didáctica en 456 estudiantes divididos en seis grupos de alumnos de cuarto, quinto y sexto grado de cinco instituciones educativas oficiales de Neiva, representa una intervención pedagógicadidáctica para fomentar gusto por la lectura y para otorgarle poder y autonomía al alumno. El apoyo teórico se acogió a las modernas concepciones de la didáctica, la práctica docente como proceso de investigación, el gusto y la lectura. La metodología estuvo orientada por prácticas docentes que permearon el diseño, la ejecución y la evaluación de la Secuencia Didáctica en su totalidad. Las conclusiones de este trabajo están referidas a la verificación de la eficacia de la Secuencia Didáctica como categoría teórico-práctica llevada al aula escolar.

Palabras claves:

Intervención pedagógica-didáctica. Secuencia didáctica. Gusto lector. Lectura.

\begin{abstract}
The researchers from the LENGUAJEANDO ("LANGUAGING") investigation group that belong to the Spanish Language Degree of the Surcolombiana University applied the Didactical Sequence as a Didactical category- in six groups of students that belong to fourth, fifth and sixth grade of 5 public education institutions of Neiva City as an educationaldidactical intervention to promote the reading enjoy. The theoretic support followed the modern ideas of didactics, the teacher practice as investigation process and the joy of reading. The methodology was guided by open, supportive and appealing teachers' practice that permeated the design, execution and evaluation of the whole Didactical Sequence. The conclusions of this research are referred to the effectiveness checking of the Didactical Sequence as a theorypractice category brought to the classroom.
\end{abstract}

\section{keywords:}

Educational-didactical intervention. Didactical Sequence. Joy reader. Reading.

\section{Zusammenfassung}

Die Forscherinnen der Forschungsgruppe LENGUAJEANDO des Studiengangs Spanische Sprache der Universidad Surcolombiana verwendeten die Didaktische Sequenzierung als didaktische Kategorie in sechs Schülergruppen der vierten, fünften und sechsten Klassenstufe an fünf offiziellen Bildungseinrichtungen Neivas, Sie benutzten sie somit als pädagogisch-didaktische Intervention für die Förderung des Interesses am Lesen.

Das theoretische Fundament schließt die modernen Auffassungen von Didaktik, die Unterrichtspraxis als Prozess der Forschung, das Interesse und die Lektüre mit ein.

Die Methodologie war an offenen, gemeinsamen und kurzweiligen Lehrpraktiken orientiert, die die Gestaltung, die Ausführung und die Beurteilung der didaktischen Sequenzierung in ihrer Gesamtheit durchdrangen.

Die Schlussfolgerungen dieser Arbeit wurden auf die Verifizierung der Effizienz der Didaktischen Sequenzierung, übertragen auf das Klassenzimmer als theoretisch-praktische Kategorie, bezogen.

1 - Magíster en Literatura. Universidad Surcolombiana. Neiva.Colombia. posadamyriam@yahoo.es

2 - Magíster en Desarrollo Educativo y Social. Universidad Surcolombiana. Neiva. Colombia. icrigu@yahoo.es

3 - Magíster en Desarrollo Educativo y Social. Universidad Surcolombiana. Neiva. Colombia. Marive1946@yahoo.es 


\section{Introducción}

Este proyecto se desarrolló durante el año 2012 en Instituciones Educativas oficiales de Neiva en los grados cuarto, quinto y sexto, y se comprobó que la promoción de la lectura necesariamente debe estar mediada por el fomento del gusto que los maestros logren inculcar en los alumnos. Además, que el maestro puede lograr sus objetivos educativos, si no se ocupa únicamente de orientar procesos lectores de comprensión en sus niveles literal, inferencial y crítico por ejemplo, del Plan Lector, sino, que es conveniente que el gusto y el placer no se restrinjan al ámbito de textos literarios, apartando al alumno de la inclinación por otras lecturas que involucran la amplia gama de la tipología textual: textos periodísticos, filosóficos, icónicos y las historietas.

El contexto teórico general en el que se ubicó la Investigación es transdisciplinario; es decir, se relacionaron conceptos y enfoques de la pedagogía, como disciplina que orienta los procesos de educabilidad, con los de la didáctica general, como discurso que media el saber disciplinario, pensando la enseñanza como objeto central de sus elaboraciones, en tanto pretende describir y explicar las prácticas docentes (Litwin: 1997); y con los de la didáctica de la lengua castellana y la literatura, concebida como el conjunto complejo de procesos de enseñanza y aprendizaje de la lengua materna y su literatura, para incidir las situaciones de aprendizaje y enseñanza (Camps: 2004), lo que implica derroteros metodológicos secuenciados desde unas prácticas y unos procesos comunicativos en el aula, la enseñabilidad de los saberes.

En este contenido teórico se concibió el Lenguaje como una práctica situada socio-históricamente, es decir, desde una dimensión social y cultural y por ello, el diseño de situaciones didácticas tiene en cuenta no sólo el desarrollo cognitivo del estudiante como criterio de organización de la enseñanza, sino de interacciones y situaciones significativas, en este caso, mediante Secuencias Didácticas para motivar gusto por la lectura. Si afirmamos que la didáctica es la disciplina que integra saberes cuando se ocupa de las prácticas de enseñanza; es la didáctica del lenguaje la que nos permite reflexionar, teorizar y diseñar las situaciones didácticas para que el estudiante se apropie del proceso y avance al aceptar el reto y la exigencia que le plantea el maestro en un trabajo colectivo de acompañamiento de los procesos implicados en la lectura.

En esta integración de saberes incluimos la lectura como práctica de libertad para la construcción de una cultura del diálogo, por cuanto uno de los derechos más preciados del ser humano es el de comunicar libremente sus pensamientos y opiniones. Por lo tanto, si concebimos la educación como diálogo, observamos que con los procesos de lectura placentera se puede generar un cambio en las relaciones maestro-alumno, lo que implica que los educandos aprendan y se eduquen, es decir, aprendan a aprender, a leer y a interpretar su propia historia para que no sean unos simples consumidores de una cultura sin sentido para ellos. Este proceso de desarrollo de la libertad de expresión es gradual en la escuela, lo que comprobamos con la ejecución de las secuencias didácticas al propiciar autonomía, para que los alumnos se formen como personas con derechos y dignidad.

Las propuestas de los autores mencionados sugieren que se privilegie el protagonismo del estudiante en el aula -como sujeto activo del proceso-; pero además, existe una responsabilidad como maestros implicados en "(...) las situaciones didácticas y las condiciones institucionales en las dificultades que "encontramos" en los alumnos". En este sentido, Paula Carlino (1999), se pregunta "¿qué tienen que ver la escritura y la lectura con el aprendizaje y la enseñanza?". Observemos que esta pregunta también ha aflorado en nuestro Grupo de investigación y en los Grupos de la Facultad de Educación.

Con estas perspectivas se tuvo en cuenta las implicaciones que tienen los nuevos soportes de la información, puesto que las prácticas de lectura se han transformado en la Escuela a medida que la sociedad acoge esas "nuevas prácticas letradas". Según Daniel Cassany "El desarrollo tecnológico y social ha generado nuevas formas de producción, transmisión y recepción de escritos. (...). Hoy los escritos tienen formas, funciones y características diferentes" (2008). Este autor también se refiere al "conflicto lingüístico" que se suscita en el aula, debido a las restricciones metodológicas en la orientación del proceso que realiza el sujeto cognoscente; en efecto, por la normativa y la instrumentación que predomina en el aprendizaje teórico-práctico de una lengua y afirma que los estudiantes están muy lejos de darse cuenta y de entender que la lengua es la que utilizan cada día para comunicarse, jugar, hablar, estudiar, aprender, etc.; que con la lengua pueden leer historias divertidas, inventar personajes o explicar chistes con gracia (Cassany, 2008).

Las prácticas pedagógicas de los maestros, orientadas a despertar el gusto por la lectura, tendrían que tener en cuenta los postulados de la moderna ciencia cognitiva, para que la pregunta por cómo es que el hombre procesa la información oriente los procesos de inducción, fomento y construcción del gusto lector, sin desconocer los factores externos que corresponden al contexto social y cultural.

En el campo teórico del gusto lector, se debe tener en cuenta las posturas lúdicas que plantea Daniel Pennac (1993) en su texto dedicado al lector y al fomento de la lectura placentera donde manifiesta "El derecho a no leer", entre otros tópicos audaces y provocadores.

Edmund Burke (2005), considera que estas disciplinas tratan el problema del gusto como una "facultad 
delicada y aérea, que parece demasiado volátil para resistir siquiera las cadenas de una definición, no se puede poner a prueba debidamente bajo ningún criterio, ni medirse por ninguna norma" (2005), sabemos que el hombre percibe el gusto con todos sus sentidos, en experiencias comunes a todas las personas; las diferencias estarían en "los hábitos, los prejuicios o los males de este hombre particular, y hemos de extraer nuestra conclusión de todo ello". Burke (2005) trata el gusto como facultad de juzgar obras de arte, como poder de la imaginación y como conocimiento superior, lo que hace pensar que tendría alcance universal en tanto el objeto estético -o bello-, es observado, comparado y definido en situaciones cotidianas, en este caso, la lectura de todo texto, lingüístico o semiótico en el aula escolar.

Relacionamos los asertos de Burke (2005), con lo que Alexander Gottieb Baumgarten (1965) nombraría como estética o ciencia del conocimiento sensorial frente a la lógica, ciencia del conocimiento cognitivo; posteriormente, Immanuel Kant (2008) designaría a la Estética como "denominación para el estudio gnoseológico de la sensación y de sus formas apriorísticas de espacio y tiempo", lo que denominó Estética trascendental, relacionando lo artístico (creación del autor) con lo sensible (recepción del espectador o del lector). También Levin L. Schücking (1996), se ocupó del tema del gusto literario con enfoque sociológico, referido al "espíritu" de una época como resultado de condiciones de "conexiones histórico-sociológicas", como también lo haría Alfred von Martin en 1931 con su amplio estudio Sociología del Renacimiento.

Esta cadena conceptual ubica el problema del gusto lector en un ámbito teórico que propicia la reflexión sobre las prácticas pedagógicas y didácticas de los maestros de los grados cuarto, quinto y sexto, por cuanto resulta complejo propiciar el gusto lector. Como ya se ha mencionado, el objeto central de la Didáctica lo constituyen las prácticas de enseñanza que toman formas diversas en atención a las concepciones, creencias y decisiones del docente. A estas formas de la práctica se les denomina configuraciones didácticas entendidas según Litwin como:

La manera particular que despliega el docente para favorecer los procesos de construcción del conocimiento. Esto implica una construcción elaborada en la que se pueden reconocer los modos como el docente aborda múltiples temas de su campo disciplinar [...], el estilo de negociación de significados que genera, las relaciones entre la práctica y la teoría que incluyen lo metódico y la particular relación entre el saber y el ignorar. Todo ello evidencia una clara intención de enseñar, de favorecer la comprensión de los alumnos y de generar procesos de construcción de conocimiento, con lo cual se distinguen claramente aquellas configuraciones no didácticas, que implican sólo la exposición de ideas o temas, sin tomar en cuenta los procesos de aprender del alumno (1997).

Los planteamientos anteriores conducen a que se conciban las secuencias didácticas a partir de los planteamientos de autores Anna Camps y Mauricio Pérez Abril (2005). Anna Camps (2004), concibe la SD como una unidad de enseñanza de la composición que se caracteriza por formular un proyecto de trabajo cuyo objetivo es la producción de un texto (oral, escrito), y que se desarrolla durante un determinado período más o menos largo, según convenga y a partir de una situación discursiva que le dará sentido, partiendo de la base que texto y contexto son inseparables. Para Pérez Abril (2005), las Secuencias Didácticas son unidades de trabajo que se ocupan de procesos y saberes muy puntuales.

En el campo escolar la lectura es un proceso interactivo entre el estudiante-lector y el texto, el lector utiliza conocimiento previo y otras estrategias para construir sentido y comprender las ideas del escritor mediante un proceso que crece y madura constantemente, y que no se aprende como una jerarquía de destrezas.

Referido al discurso narrativo, Mijaíl Bajtín (1992), formuló la teoría de los géneros discursivos desde la década de los 50 del siglo XX, concibiéndolos como:

Formas discursivas convencionales conformadas históricamente en una cultura determinada. Se van constituyendo a lo largo de la historia de una comunidad lingüística como prácticas sociales con valor funcional. Son, por lo tanto, productos socioculturales y como tales se reconocen fácilmente entre los miembros de una comunidad. Son hechos comunicativos que suceden en un contexto social, de acuerdo con ciertas normas y convenciones, y con una clara finalidad, por ejemplo, la de informar, persuadir, divertir, etc.

Hoy, después de cuatro décadas de reflexión teórica sobre la clasificación de los géneros discursivos no hay unanimidad, pero propuestas como la de Werlich (1979) propone una tipología basada en las estructuras cognitivas, es decir, según los modos de percibir la realidad (dimensión cognitiva) y según los modos de representar la realidad (dimensión lingüística). Adam (1992), recoge y elabora esta teoría y propone la clasificación pero a partir de secuencias textuales prototípicas, pues admite el texto como un conjunto de secuencias de varios tipos que se articulan entre sí y se van alternando.

\section{Metodología}

Se seleccionó la Secuencia Didáctica como posibilidad de integrar teoría y práctica en un proceso de intervención didáctica, concibiendo la SD como una estructura de acciones e interacciones intencionales y relacionadas entre sí, organizadas para alcanzar algún tipo de aprendizaje, como recomienda Gloria Rincón (2004). Además, la SD permitió identificar, tanto sus propósitos y condiciones de inicio, desarrollo y cierre, como los procesos y resultados. Es decir, la SD como hipótesis de trabajo que permitió la construcción, deconstrucción y reconstrucción del proceso de enseñanza-aprendizaje, de acuerdo con la realidad del aula escolar intervenida; así, en el Informe damos cuenta detallada de cómo ocurrieron las cinco sesiones dedicadas al desarrollo de cada SD.

Además de circunscribir el análisis de los resultados de la ejecución de las Secuencias Didácticas en los grados ya señalados, se buscó sugerir caminos para realizar un trabajo permanente con las docentes y 
directivas de cada Institución Educativa, para lograr que más niños, jóvenes y adultos encuentren verdadero placer y sentido vital en la lectura, desde su propia actitud satisfactoria. La planeación, la aplicación y la evaluación de dos secuencias didácticas tuvo como objetivo general contribuir al desarrollo del gusto lector de alumnos de las Instituciones educativas oficiales de la ciudad de Neiva. Las SD correspondieron al trabajo con el discurso narrativo icónico "Un encuentro con la historieta" y con el discurso narrativo artístico "Descubriendo con los niños las aventuras de don Quijote".

Se proyectaron cinco (5) sesiones para el desarrollo de los contenidos de las dos SD. Cada sesión se definió como:

Una unidad de análisis de menor amplitud que la unidad de Secuencia Didáctica, en las que se explicitan procedimientos mediante los cuales, los participantes construyen conocimiento mediante un trabajo cooperativo y acuerdan el camino a seguir sobre significados y experiencias, que les permite construir significados compartidos. Coll (1995).

Las Secuencias Didácticas estuvieron constituidas por los siguientes elementos: Número de sesión y fecha; nombre de la sesión; objetivos; contenidos conceptuales, procedimentales y actitudinales; recursos; evaluación y resultados esperados. Cada una de las actividades propuestas estuvo enmarcada en la intencionalidad didáctica del aprendizaje significativo, para que el estudiante fuera protagonista autónomo de su aprendizaje; es decir, se apropiara de su saber construido por él y para él, y que no lo considerara como una imposición o un deber.

En este sentido, destacamos la colaboración de la población beneficiada que consistió en quince docentes del área de Lengua Castellana y cuatrocientos cincuenta y seis (456) estudiantes de las Instituciones Educativas involucradas en el proceso, lo que corresponde a un porcentaje del $38 \%$ sobre mil setecientos treinta (1.730), que es el total de la población.

Un elemento fundamental durante el proceso de sistematización de la Secuencia Didáctica fue el conjunto de instrumentos creados para posibilitar la interpretación de los procesos durante el desarrollo de las Secuencias "Un encuentro con la Historieta" y "Descubriendo con los niños las aventuras de don Quijote".

A continuación listamos los diferentes instrumentos que se implementaron en las aulas escolares, acordes con el diseño metodológico y las categorías de análisis:

Reconocimiento de saberes previos

Portafolio del estudiante

Portafolio del profesor

Diario de campo

Instrumento final de Sistematización

\section{Conclusiones}

La planeación y el desarrollo de las Secuencias Didácticas "Un encuentro con la Historieta" y "Descubriendo con los niños las aventuras de don Quijote", estimularon el trabajo conjunto de los actores del proceso, en este caso la maestra y los estudiantes principalmente, además de la participación de otros actores de la comunidad educativa como los docentes titulares de la asignatura, los directivos de la Institución Educativa y los padres de familia. Esto evidencia que la SD como estrategia pedagógica es una opción significativa y pertinente para despertar el gusto por la lectura de diversos géneros discursivos, porque replantea las prácticas pedagógicas y convierte a los niños y niñas en los protagonistas de los procesos de construcción de saberes, es decir, se privilegia el aprendizaje al empoderar significativamente a la población estudiantil como constructor de su aprendizaje.

En este mismo sentido William Ospina en la obra Los nuevos centros de la Esfera (2001), afirma que "Si alguna revolución requiere la educación pienso que es la revolución de la alegría, que les devuelva o les confiera a los procesos educativos su radical condición de aventura apasionada, de expedición excitante, de juego y de fiesta." A partir de la afirmación anterior se elaboró y desarrolló propuesta de Secuencia Didáctica basada en la versión infantil de la obra clásica El Quijote de la Mancha, como posibilidad de acercamiento al disfrute de una obra clásica con alto sentido estético, lenguaje lúdico y jocoso. Como lo plantea Ítalo Calvino, se contribuyó a recuperar desde la Escuela la lectura acompañada de los clásicos, teniendo en cuenta su aporte cultural y su trascendencia, la influencia particular que ejercen, cuando se imponen por inolvidables, o cuando se esconden en los pliegues de la memoria mimetizándose con el inconsciente colectivo o individual.

Otro alcance fundamental de la propuesta desarrollada fue la de forma como se logró cambiar la concepción que se tenía frente a la enseñanza del lenguaje a través de la democratización de la voz en el aula, puesto que se propició el empoderamiento del estudiante frente a su aprendizaje y al proceso de enseñan$\mathrm{za}$, a partir de los cambios que se operaron en la recontextualización de las prácticas de enseñanzaaprendizaje, al implementar las Secuencias Didácticas.

La selección de los textos para el fomento del gusto lector son de gran importancia, puesto que en el caso del género narrativo representado en un texto como la historieta y los elementos que la componen -imágenes, textos cortos, onomatopeyas, viñetas etc.-, permiten facilitar la compresión del significado y sentido. De la misma forma el género narrativo literario basado en la obra El Quijote de la Mancha, despertó el interés por este tipo de textos de la literatura clásica universal. 
Otro de los logros centrales fue el reconocimiento del estudiante como un agente activo del acto pedagógico representado en la participación en los procesos del aula, en donde se hace un reconocimiento de su voz y de la de sus pares al respetar la palabra y sus ideas, en donde se ejerce una verdadera democracia en su formación como sujeto social crítico del entorno y de su mundo. Finalmente, se reitera cómo los procesos de enseñanza de la lectura en la Escuela han de orientarse como una actividad placentera, que se convierta en una herramienta para la vida. Es decir, formar niños y jóvenes deliberativos, con capacidad para expresar en forma argumentada posiciones claras y posturas críticas respecto a los fenómenos sociales, culturales, políticos, entre otros, que ocurren en su entorno globalizado y atomizado de información.

\section{Referencias Bibliográficas}

Adam, J.M. Secuencias textuales prototípicas. París: 1992. Bajtín, Mijail (1998). Estética de la creación verbal. México: Siglo XXI Baumgarten, A. G. en Reflexiones filosóficas acerca de la poesía. Consulta en: http://es.wikipedia.org/wiki/Alexander_Gottlieb_Baumgarten Burke, Edmund (2005). De lo sublime y de lo bello, Madrid, Alianza Editorial.
Calsamiglia BLANCAFORT, Helena y TUSÓN VALLS, Amparo (1999): Las cosas del decir, Barcelona, Ariel.

Camps, Anna (2004). "Objeto, modalidades y ámbitos de la investigación en didáctica". En: Lenguaje, No 32, Universidad del Valle, p. 7-27.

Camps, Anna (compiladora) (2003). Secuencias Didácticas para aprender a escribir. Barcelona: Graó.

Carlino, P. y SANTANA, (eds.) (1999). Leer y escribir con sentido: una experiencia constructivista en educación infantil y primaria. Barcelona: Visor.

Carlino, Paula (2006). Escribir, leer y aprender en la universidad, Buenos Aires, Fondo de Cultura Económica de Argentina (material policopiado como material didáctico)

Cassany, Daniel (2008). Prácticas Letradas Contemporáneas, México, Sevilla Editores S. A. Colección Ríos de Tinta.

Cassany, Daniel y otros (2005). Enseñar lengua, Barcelona, Editorial Graó.

Kant, Immanuel (2008), Observaciones acerca del sentimiento de lo bello y de lo sublime, Madrid, Alianza Editorial.

Litwin, Edith (1997). Las Configuraciones Didácticas: Una nueva agenda para la enseñanza superior. Buenos Aires: Paidós.

Litwin, Edith (1997). Las Configuraciones Didácticas: una Nueva Agenda para la Enseñanza Superior, Buenos Aires, Paidós.

Pennac, Daniel (1993). Como una novela, Bogotá, Editorial Norma.

Pérez, Abril Mauricio (2005). "Un marco para pensar Configuraciones Didácticas en el campo del Lenguaje, en la Educación Básica". En: Investigar la lectura y la escritura en el aula. Buga: Universidad del Valle.

Pérez, Mauricio Y RINCÓN, Gloria (2009). Actividad, Secuencia Didáctica y Pedagogía por Proyectos. Tres alternativas para la organización del trabajo didáctico en el campo del lenguaje. Bogotá: CERLALC.

Schücking, Levin L. (1996). El gusto literario, México, Fondo de Cultura Económica, quinta reimpresión (1931). 